\title{
Nano Ferric Oxide: Synthesis, Characterization and Catalytic Activity on Thermal Decomposition of Ammonium Perchlorate
}

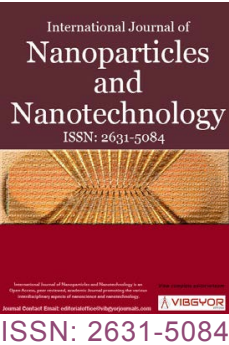

\section{Yehia*}

School of Chemical Engineering, Military Technical College, Kobry El-Kobba, Cairo, Egypt

\begin{abstract}
A lot of attention has been given to incorporation of Nano-sized oxides including $\mathrm{Fe}_{2} \mathrm{O}_{3}$ in ammonium perchlorate (AP) based solid propellants to enhance their final performance. Nano ferric oxide was synthesized by a solid-state reaction method. The obtained products were characterized by scanning electron microscope (SEM), transmission electron microscopy (TEM) and X-ray diffraction (XRD) for particle size, purity and morphology. Size reduction of the catalyst increases the surface area and hence, the catalytic activity is also increased. The thermal behavior of AP in the presence of Nano-ferric oxide was studied by using differential scanning calorimetry (DSC). It was found that the average particle size of Nano-ferric oxide prepared was in the range of $10-20 \mathrm{~nm}$. Interestingly, catalytic activity of AP where the rate of thermal decomposition of AP in the presence of $1 \%$ Nano-ferric oxide increased $10 \%$, which have a direct effect on the burning behavior of propellants.
\end{abstract}

\section{Introduction}

At Nano scale, the physicochemical characteristics of materials are considerably various from those found in larger scales and bulk materials [1]. Nanosized particles, due to small particle size, large surface area, many surface atoms complex microstructures, and defects of grain, have high catalytic activity. therefore, replacing the conventional catalysts in solid propellant by Nano-sized catalysts becomes a key researching hot point to improve the combustion performance of propellants [2,3]. Metal oxides are an important class of chemicals having a wide range of applications in many areas of chemistry, physics and material science [4]. Metal oxide Nano crystals can exhibit unique physico-chemical properties due to their Nano size and high density of cover or edge surface sites [5]. Composite solid propellants (CSPs) are the major source of chemical energy in space vehicles and missiles. Ammonium perchlorate (AP) is widely used as an oxidizer in CSPs $[6,7]$. The ballistics of a composite propellant can be improved by adding a catalyst, which accelerates the rate of decomposition of AP $[8,9]$. Recent investigations have shown that nanoparticles of transition metal oxides (TMOs), without any agglomeration can increase the burning rate of propellants [10]. The efficiency of catalytic action increases sharply in Nano sized oxide particles than micro scale oxide particles [11,12].

In this paper, Nanometer-sized $\mathrm{Fe}_{2} \mathrm{O}_{3}$ synthesis by a solid-state reaction method and study catalytic activity of Nanometer-sized $\mathrm{Fe}_{2} \mathrm{O}_{3}$ on thermal decomposition of ammonium perchlorate, which

*Corresponding author: M Yehia, School of Chemical Engineering, Military Technical College, Kobry El-Kobba, Cairo, Egypt

Accepted: December 26, 2018; Published: December 28, 2018

Copyright: (C) 2018 Yehia M. This is an open-access article distributed under the terms of the Creative Commons Attribution License, which permits unrestricted use, distribution, and reproduction in any medium, provided the original author and source are credited.

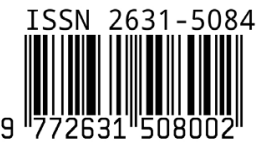

Yehia. Int J Nanoparticles Nanotech 2018, 4:020 
have a direct effect on the burning behaviors of propellant.

\section{Experimental Work}

\section{Materials}

All the reagents were analytical-grade chemicals. $\mathrm{Fe}\left(\mathrm{NO}_{3}\right)_{3} \cdot 9 \mathrm{H}_{2} \mathrm{O}, \mathrm{NaOH}$ and ethanol were purchased from (sigma Aldrich), and Poly (acrylic acid) (PAA) with an average molecular weight (Mw) of $1600 \mathrm{~g}$ $\mathrm{mol}^{-1}$ was employed as polymeric surfactant, was purchased from Morgan company (Cairo, Egypt).

\section{Synthesis of nano ferric oxide}

First, $0.02 \mathrm{~mol} \mathrm{Fe}\left(\mathrm{NO}_{3}\right)_{3} .9 \mathrm{H}_{2} \mathrm{O}, 0.06 \mathrm{~mol} \mathrm{NaOH}$ and around $0.4 \mathrm{ml}$ (PAA) used as dispersant were put in a batch reactor at temperature $300{ }^{\circ} \mathrm{C}$ for about 30 minutes. The synthesized colloidal particles demonstrated deep red color. The particles flocculate with $30 \mathrm{~min}$. Subsequently, the products were washed with distilled water and treated in an ultrasonic bath with absolute ethanol.

\section{Characterization of Synthesized Nano Ferric Oxide}

The size and shape of synthesized colloidal nanoparticle were investigated using TEM (JEM$2100 \mathrm{~F}$ by Joel Corporation). The crystalline phase was investigated with XRD, XTRA Powder diffractometer by Thermo Scientific, over the angle range from 5 to $80{ }^{\circ} \mathrm{C}$. The dry powder size and shape were investigated with SEM, ZEISS SEM EVO $10 \mathrm{MA}$, with three types of detectors secondary electrons (SE), back scattered electron (BSE), and energy dispersive X-ray spectrometer (EDX) Bruker Quanta 200. Nanometer-sized $\mathrm{Fe}_{2} \mathrm{O}_{3}$ and AP were mixed in a 1:99 (wt :\%) ratio to prepare the samples for the thermal analyses experiment. The thermal decomposition of AP was investigated by differential scanning calorimetry (DSC) (Q20) with the heating rate of $10^{\circ} \mathrm{C} / \mathrm{min}, \mathrm{N}_{2}$ atmosphere, the temperature ranges from $20{ }^{\circ} \mathrm{C}$ to $500{ }^{\circ} \mathrm{C}$ and the sample weight was $1.0 \mathrm{mg}$.

\section{Result and Discussions}

\section{TEM characterization}

TEM micrographs of synthesized Nano ferric oxide demonstrated mono-dispersed particles of 10-20 nm average particle size (Figure 1a). The diffraction of the incident beam demonstrated mono-crystalline structure (Figure 1b).

\section{XRD characterization}

Nano ferric oxide particles were dried. XRD diffractogram of synthesized Nano ferric oxide demonstrated pure crystalline structure of $\mathrm{Fe}_{2} \mathrm{O}_{3}$ (Figure 2). All characteristic peaks of $\mathrm{Fe}_{2} \mathrm{O}_{3}$ shown in (Figure 2) agreed with the Joint Committee on Powder Diffraction Standards (JCPDS). The particle size of ferric oxide might be estimated by the Scherrer equation [13] which is $10 \mathrm{~nm}$.

\section{SEM characterization}

Nano ferric oxide particles were dried. SEM micrographs of synthesized Nano ferric oxide demonstrated polls of Nano ferric oxide particles like cotton flower but the particles still separately from each other (Figure 3). This means that Nano ferric oxide particles still keep their large surface area and there aren't any bonds between the particles. The Nano ferric oxide particles can be
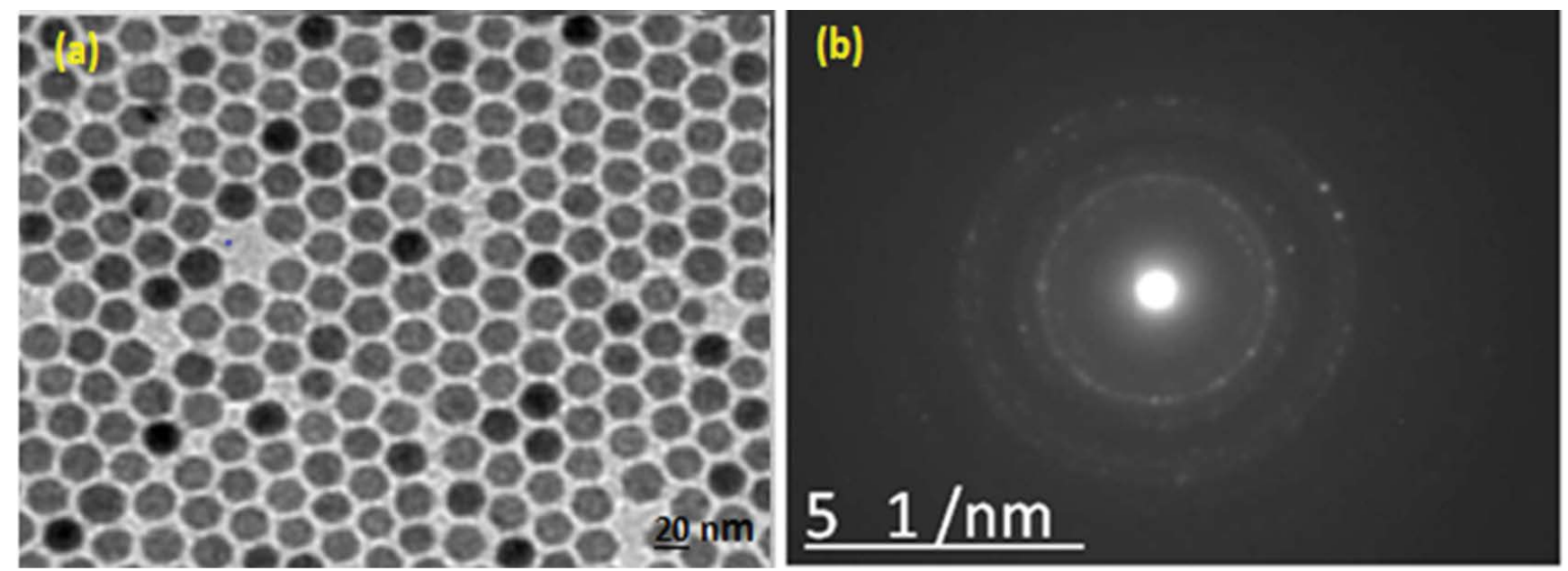

Figure 1: TEM micrographs of synthesized Nano ferric oxide. 


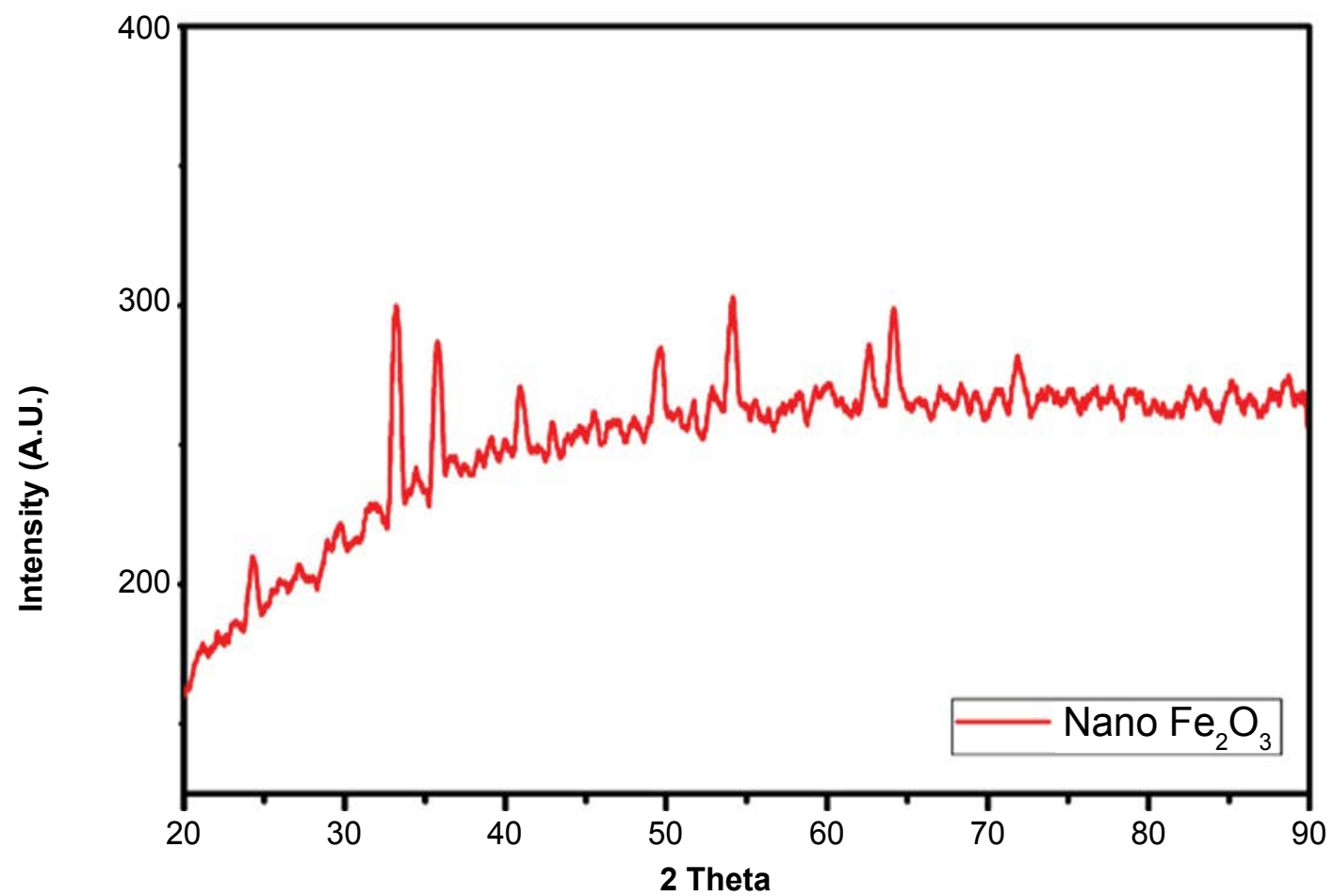

Figure 2: XRD pattern of synthesized Nano ferric oxide.
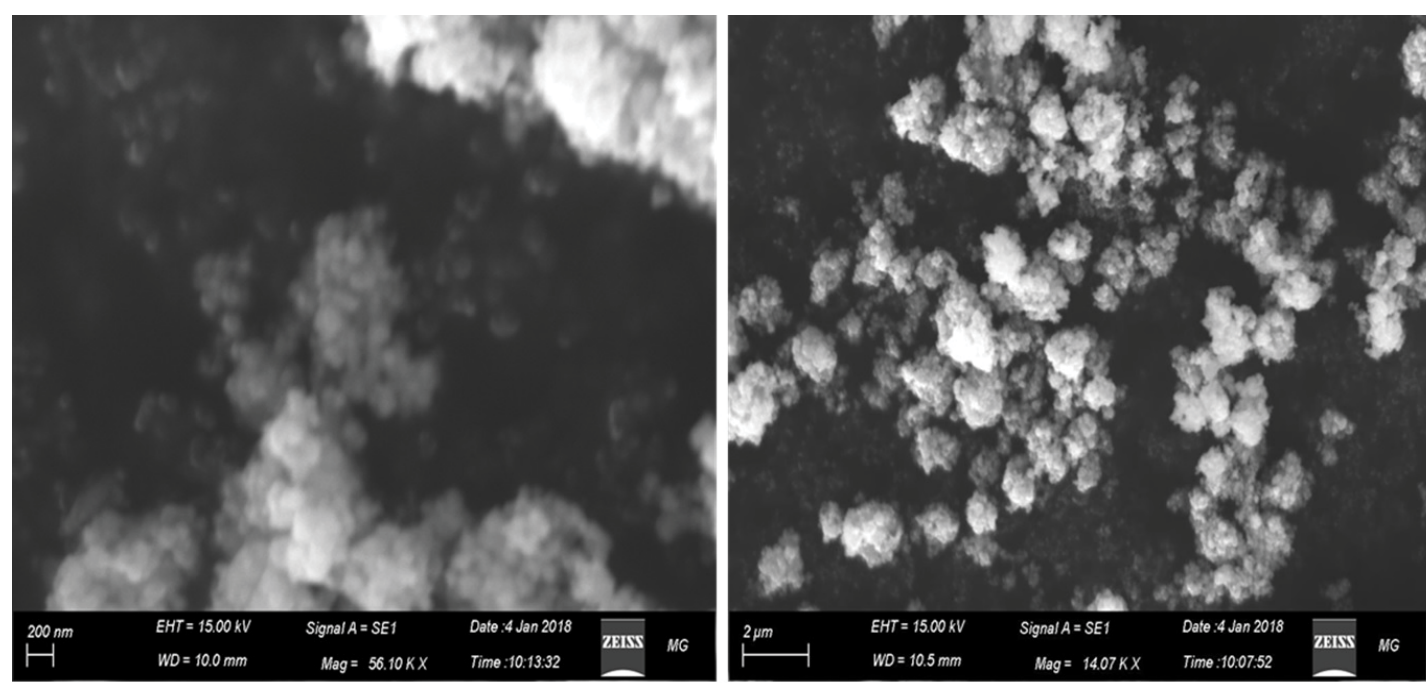

Figure 3: SEM micrographs of synthesized Nano ferric oxide.

easily re-dispersion by sonicate into a solvent.

\section{Catalytic activity on the thermal decomposi- tion of AP}

DSC curves for the thermal decomposition of pure AP and AP mixed with Nano ferric oxide in a 1:99 (wt :\%) are illustrated in (Figure 4). Analysis showed that the thermal decomposition of pure AP particles occurs in two stages: the endothermic stage and the exothermic stage [14]. The endothermic stage happens at a temperature of about $240{ }^{\circ} \mathrm{C}$. This stage could be attributed to the crystal phase transition from orthorhombic to cubic. The other two peaks are representing the exothermic peaks; the first peak appears at 270 ${ }^{\circ} \mathrm{C}$ for the partial decomposition of $\mathrm{AP}$ and the formation of some $\mathrm{NH}_{3}$ and $\mathrm{HClO}_{4}$ via dissociation and sublimation. The second peak appears at 450 ${ }^{\circ} \mathrm{C}$ for complete decomposition of AP and thus formation of volatile product.

The thermal decomposition of AP is sensitive to Nano ferric oxide. The DSC curve of the 


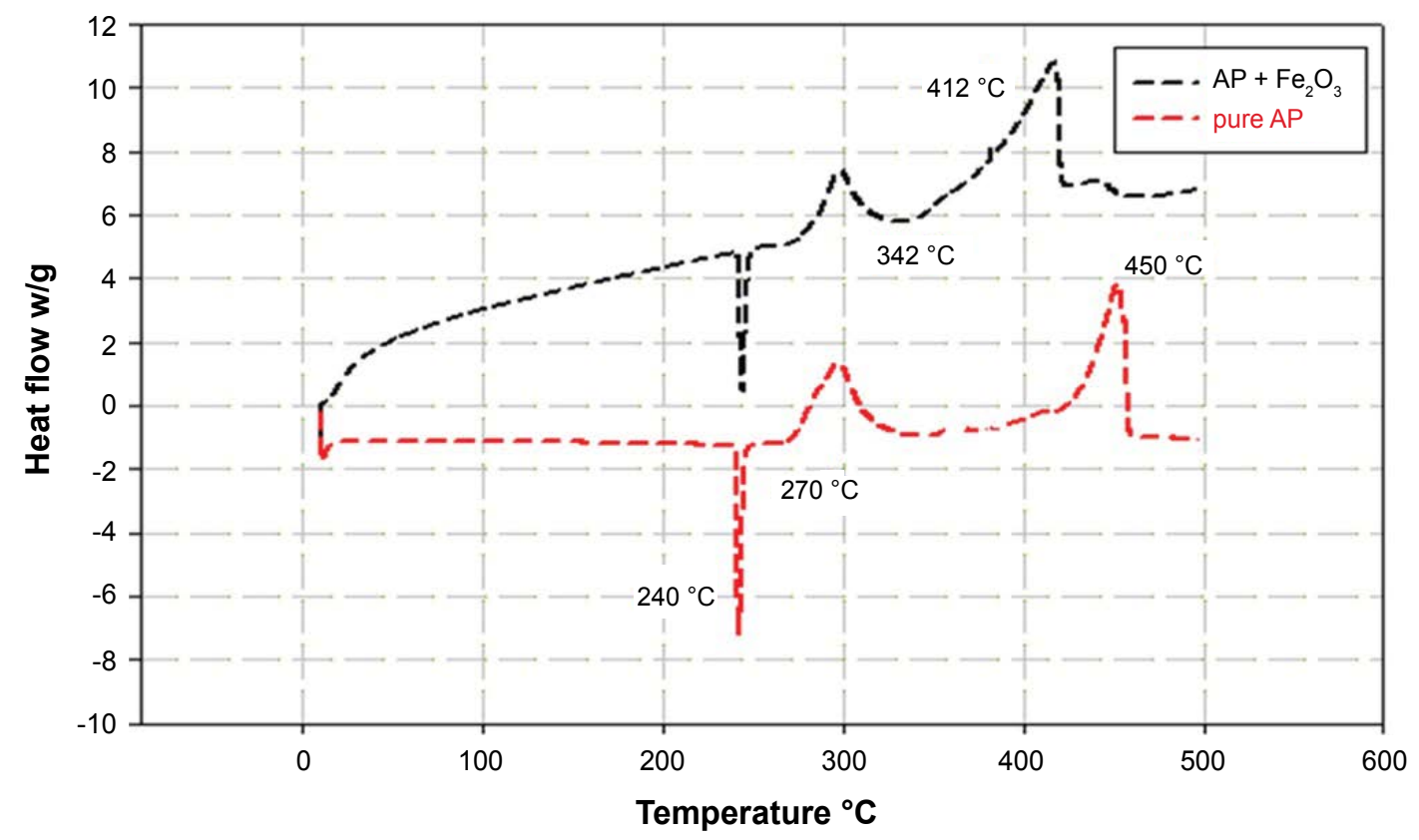

Figure 4: DSC curves of pure AP and $A P+\mathrm{Fe}_{2} \mathrm{O}_{3}$.

decomposition of AP with Nano ferric oxide additives obvious that addition of Nano ferric oxide to AP has no deep effect on its crystallographic phase transition temperature. On the other hand, it has considerable influence on the exothermic decomposition of AP. The first exothermic peak of $\mathrm{AP}+\mathrm{Fe}_{2} \mathrm{O}_{3}$ appears at the same temperature of pure AP at $270{ }^{\circ} \mathrm{C}$, while the second exothermic peak start appear at much lower temperature of $342{ }^{\circ} \mathrm{C}$. This indicates that the catalytic activity of Nano ferric oxide on the Thermal Decomposition of AP, which has a direct effect on the burning behaviors of propellant formulations.

\section{Conclusion}

The preparation of Nano ferric oxide was successfully done using solid state method.

The average particle size of Nano-ferric oxide prepared was in the range of $10-20 \mathrm{~nm}$. Nano ferric oxide was catalytically active for the thermal decomposition of AP. the rate of thermal decomposition of AP in the presence of $1 \%$ Nano ferric oxide increased $10 \%$, which have a direct effect on the burning behavior of propellants.

\section{References}

1. IM El-Sherbiny, E Salih, FM Reicha (2013) Green synthesis of densely dispersed and stable silver nanoparticles using myrrh extract and evaluation of their antibacterial activity. Journal of Nanostructure in Chemistry 3: 8.
2. LT DeLuca, L Galfetti (2008) Burning of metallized composite solid rocket propellants: From micrometric to nanometric aluminum size. Proceedings $3^{\text {rd }}$ Asian Joint Conference on Propulsion and Power (AJCPP).

3. V Babuk, I Dolotkazin, A Gamsov, A Glebov, LT DeLuca, et al. (2009) Nanoaluminum as a solid propellant fuel. Journal of Propulsion and Power 25: 482-489.

4. C Nogeura (1996) Physics and chemistry at oxide surface. Cambridge University Press, Cambridge, UK.

5. C Feldmann, HO Jungk (2001) Polyol-mediated preparation of nanoscale oxide particle. Angewandte Chemie International Edition 40: 359-362.

6. PWM Jacobs, $H$ Whitehead (1969) Decomposition and combustion of ammonium perchlorate. Chemical Reviews 69: 551-590.

7. V Boldyrev (2006) Thermal decomposition of ammonium perchlorate. Thermochimica Acta 443: 1-36.

8. S-M Shen, S-I Chen, B-H. Wu (1993) The thermal decomposition of ammonium perchlorate (AP) containing a burning-rate modifier. Thermochimica acta 223: 135-143.

9. J Gao, F Guan, Y Zhao, W Yang, Y Ma, et al. (2001) Preparation of ultrafine nickel powder and its catalytic dehydrogenation activity. Materials Chemistry and Physics 71: 215-219.

10. VS Sedoi, YF Ivanov, MN Osmonoliev (2003) Study of ultra-fine powders produced by the exploding 
wire method. $34^{\text {th }}$ International Annual Conference of ICT 1-13.

11. M Telkar, C Rode, R Chaudhari, S Joshi, A Nalawade (2004) Shape-controlled preparation and catalytic activity of metal nanoparticles for hydrogenation of 2-butyne-1, 4-diol and styrene oxide. Applied Catalysis A: General 273: 11-19.

12. SS Joshi, PR Patil, V Krishnamurthy (2008) Thermal decomposition of ammonium perchlorate in the presence of nanosized ferric oxide. Defence Science Journal 58: 721-727.

13. T Tillotson, L Hrubesh, R Simpson, R Lee, R Swansiger, et al. (1998) Sol-gel processing of energetic materials. Journal of Non-Crystalline Solids 225: 358-363.

14. M Mahinroosta (2013) Catalytic effect of commercial nano-CuO and nano- $\mathrm{Fe}_{2} \mathrm{O}_{3}$ on thermal decomposition of ammonium perchlorate. Journal of Nanostructure in Chemistry 3: 47. 